\title{
INFLUENCE OF HORIZONTAL INHOMOGENEITY IN THE IONOSPHERE ON THE REFLECTION OF ALFVÉN WAVES
}

\author{
L. Zhu, J. J. Sojka, R. W. Schunk, and D. J. Crain \\ Center for Atmospheric and Space Sciences, Utah State University
}

Abstract. A study of the reflection of Alfvén waves at a horizontally inhomogeneous ionosphere has been carried out. In this study, the Alfvén speed above the ionosphere is assumed to be uniform and the ionosphere is treated as a height-integrated conducting slab. Analytical and numerical results indicate that the horizontal nonuniformity of the ionospheric conductivity can lead to a rotation of the reflected wave fields and cause field-aligned currents that originate in the ionosphere. A strong conductivity nonuniformity in the direction perpendicular to the incident wave field, large Hall to Pedersen conductivity ratios, and low conductivity values lead to a large rotation of the reflected wave field in the range from a few to $40^{\circ}$. The implications of the theoretical results for some ionospheric phenomena are presented, including comments on the Harang discontinuity and Sun-aligned arcs.

\section{Introduction}

The reflection of Alfvén waves at the ionosphere is a key element in the study of the magnetosphere-ionosphere (M-I) coupling by means of Alfvén waves and also a fundamental issue of space plasma physics. The reflection of the Alfvén waves with a constant speed at a homogeneous ionosphere has been extensively studied [e.g., Mallinckrodt and Carlson, 1978]. In these cases, the reflection coefficient is merely determined by the ionospheric height-integrated Pedersen conductivity and the Alfvern conductivity and is independent of wave frequency. By including the nonuniformity of the Alfvén speed above the ionosphere, it was found that the Alfvén wave reflection coefficient can become dependent on wave frequency and the wave functions for the Alfvén waves can be Bessel function solutions instead of simple sinusoidal waves for the case of constant Alfvén speed [Lysak, 1991]. Most recently, a study of the influence of vertical inhomogeneity in the ionosphere on the reflection of Alfvén waves was conducted by Knudsen et al. [1992]. Their results show that the reflection features become frequency-dependent due to the vertical inhomogeneity, but for low-frequency Alfvén waves the vertically inhomogeneous ionosphere still behaves as a thin conducting slab.

It is the purpose of this work to study the detailed features of Alfvén wave reflection at a horizontally inhomogeneous ionosphere by assuming a constant Alfvén speed and a heightintegrated ionosphere. The latter assumption means that our results are limited to the low-frequency Alfvén waves. It will be shown from both analytical and numerical results that the horizontal nonuniformity of the ionospheric conductivity can lead to new features of Alfvén wave reflections that may have important ionospheric implications.

\section{Copyright 1993 by the American Geophysical Union}

Paper Number 93GL00079

0094-8534/93/93GL-00079\$03.00

\section{Basic Equations}

The field-aligned currents carried by the incident and reflected Alfvén waves can be derived from the ideal MHD equation [Alfvén and Falthammar, 1963], which is

$$
\mathbf{J}_{\| \mathrm{A}}=\mathbf{b}_{\mathrm{o}} \Sigma_{\mathrm{A}} \nabla \cdot\left(\mathbf{E}^{\mathrm{i}}-\mathbf{E}^{\mathbf{r}}\right)
$$

where $\mathbf{b}_{\mathbf{0}}$ is the unit vector of the background magnetic field, $\Sigma_{\mathrm{A}}=\left(\mu_{\mathrm{o}} V_{A}\right)^{-1}$ is the Alfvén conductivity, $\mathbf{E}^{\mathrm{i}}$ and $\mathbf{E}^{\mathrm{r}}$ are the incident and reflected Alfvén wave fields, respectively. When the ionosphere is treated as a height-integrated slab, the current continuity in the ionosphere can be expressed as:

$$
\mathbf{J}_{\|_{i}}=\nabla \bullet\left(\Sigma_{\mathrm{P}} \mathbf{E}_{\mathrm{i}}+\Sigma_{\mathrm{H}} \mathbf{b}_{\mathrm{o}} \times \mathbf{E}_{\mathrm{i}}\right)
$$

and

$$
E_{i}=E^{i}+E^{r}+E_{0}
$$

where $\mathbf{E}_{\mathbf{i}}$ is the total ionospheric electric field, $\mathbf{E}_{\mathbf{0}}$ is the background ionospheric electric field, and $\Sigma_{\mathrm{P}}$ and $\Sigma_{\mathrm{H}}$ are the ionospheric height-integrated Pedersen and Hall conductivities, respectively.

By matching the field-aligned currents carried by the incident and reflected Alfvén waves and the field-aligned current associated with the background electric field with the divergence of the total ionospheric horizontal current, we obtain the basic equation which describes the reflection of an Alfvén wave from a horizontally inhomogeneous heightintegrated ionosphere,

$$
\begin{aligned}
& \left(\Sigma_{\mathrm{A}}+\Sigma_{\mathrm{P}}\right) \nabla \cdot \mathbf{E}^{\mathrm{r}}+\nabla \Sigma_{\mathrm{P}} \cdot \mathbf{E}^{\mathrm{r}}+\nabla \Sigma_{\mathrm{H}} \cdot \mathbf{b}_{\mathrm{O}} \times \mathbf{E}^{\mathrm{r}} \\
& =\left(\Sigma_{\mathrm{A}}-\Sigma_{\mathrm{P}}\right) \nabla \cdot \mathbf{E}^{\mathrm{i}}-\nabla \Sigma_{\mathrm{P}} \cdot \mathbf{E}^{\mathrm{i}}-\nabla \Sigma_{\mathrm{H}} \bullet \mathbf{b}_{\mathrm{O}} \times \mathbf{E}^{\mathrm{i}}
\end{aligned}
$$

or it can be expressed by scalar potential

$$
\begin{aligned}
& \left(\Sigma_{\mathrm{A}}+\Sigma_{\mathrm{P}}\right) \Delta \phi^{\mathrm{r}}+\nabla \Sigma_{\mathrm{P}} \cdot \nabla \phi^{\mathrm{r}}+\nabla \Sigma_{\mathrm{H}} \cdot b_{\mathrm{o}} \times \nabla \phi^{\mathrm{r}} \\
& =\left(\Sigma_{\mathrm{A}}-\Sigma_{\mathrm{P}}\right) \Delta \phi^{\mathrm{i}}-\nabla \Sigma_{\mathrm{P}} \cdot \nabla \phi^{\mathrm{i}}-\nabla \Sigma_{\mathrm{H}} \cdot b_{\mathrm{O}} \times \nabla \phi^{\mathrm{i}}
\end{aligned}
$$

Based on equations (3) to (4), several Alfvén wave reflection features associated with the horizontal nonuniformity of the ionospheric conductivity can be qualitatively elucidated as follows:

When the ionosphere is homogeneous, it has been shown that the reflected wave field and the incident wave field are always coplanar [cf. Miallinckrodt and Carlson, 1978]. The situation can be different when the ionosphere is horizontally inhomogeneous. Assuming that $\phi^{\mathrm{i}}$ is only a function of $\mathrm{X}$ and 
that the conductivity distribution is a function of both $\mathrm{X}$ and $\mathrm{Y}$, equation (4) indicates that $\phi^{r}$ can be a function of both $X$ and $Y$. This means that the reflected wave field is no longer coplanar with the incident wave field, and the horizontal inhomogeneity of the ionosphere forces the reflected wave field to rotate to satisfy the current continuity.

Another new feature of the Alfvén wave reflection associated with the ionospheric horizontal inhomogeneity is the field-aligned current that originates in the ionosphere. This feature can be demonstrated in the following way: Assuming a uniform incident wave field, it can be seen from equation (3) that the reflected wave field must be uniform when the ionosphere is homogeneous. In this case, the magnetosphere supplies an electric field to the ionosphere, but does not provide a current source. The total ionospheric electric field resulting from the incident and reflected waves only drives a uniform horizontal current, which is of ionospheric origin and cannot be converted into a field-aligned current. When including the ionospheric horizontal inhomogeneity, equation (3) indicates that the reflected wave can be nonuniform and a field-aligned current can be associated with the reflected wave. Since the magnetosphere still does not supply a current source to the ionosphere, the field-aligned current carried by the reflected wave must be a field-aligned current that originates in the ionosphere.

More detailed features of Alfvén wave reflection at a horizontally inhomogeneous ionosphere are shown quantitatively in the following section.

\section{Reflections of Alfvén Waves}

A Cartesian coordinate system is adopted in which the ionospheric slab is in the $\mathrm{X}-\mathrm{Y}$ plane and the magnetic field points toward the $-Z$ direction. The scalar potential of the incident wave field is assumed to be a function of $\mathrm{X}$ only, therefore, $\mathbf{E}^{\mathrm{i}}$ is in the $\mathbf{X}$ direction. The angle between the $\mathbf{X}$ axis and the conductivity gradient is denoted by $\psi$. In the small region under consideration, the ratio between the Hall and Pedersen conductivities is assumed to be constant in space. As indicated by the equations in the previous section, the important factors influencing Alfvén wave reflection at a horizontally inhomogeneous ionosphere are the Alfvén conductivity $\Sigma_{\mathrm{A}}$, the integrated Pedersen conductivity $\Sigma_{\mathrm{P}}$, the gradient of the integrated Pedersen conductivity $\nabla \Sigma_{P}$, the angle between the conductivity gradient and the incident wave field $(\psi)$, and the ratio between the Hall and Pedersen conductivities (R). In the following, the Alfvén conductivity will remain constant and we will focus on the influences of the other four factors.

\section{1. $\nabla \Sigma_{P}=0$}

In the case of a homogeneous ionosphere, the field-aligned currents are closed by the Pedersen currents only and the solution to equation (3) is known [cf. Mallinckrodt and Carlson, 1978],

$$
\mathrm{E}^{\mathrm{r}}=\frac{\Sigma_{\mathrm{A}}-\Sigma_{\mathrm{P}}}{\Sigma_{\mathrm{A}}+\Sigma_{\mathrm{P}}} \mathrm{E}^{\mathrm{i}}
$$

where $\mathrm{E}^{\mathrm{i}}$ can be any function of $\mathrm{X}$.
The reflection feature indicated by equation (5) can be summarized as follows: When $\Sigma_{\mathrm{P}}>\Sigma_{\mathrm{A}}$, the reflected wave field $\mathrm{E}^{\mathrm{r}}$ is opposite in direction to the incident wave field $\mathrm{Ei}$. When $\Sigma_{\mathrm{P}}<\Sigma_{\mathrm{A}}, \mathrm{E}^{\mathrm{r}}$ and $\mathrm{E}^{\mathrm{i}}$ are in the same direction. In the above case, the conditions in the magnetosphere and ionosphere are unmatched, and therefore, a reflected wave carrying the ionospheric information is needed to tell the magnetosphere to make a further adjustment. When $\Sigma_{\mathrm{P}}=\Sigma_{\mathrm{A}}$ there is no reflected wave. In this case, the conditions in the magnetosphere and ionosphere are matched, and there is no further adjustment in the coupled M-I system. Note that in the above we have assumed there is no conductivity enhancement caused by precipitation, otherwise the change of conductivity can launch a secondary upward propagating Alfvén wave which can complicate this picture of the M-I coupling.

\section{2. $\nabla \Sigma_{P} \neq 0, \psi=0^{\circ}\left(\right.$ or $\left.180^{\circ}\right)$}

When a conductivity gradient is along the direction of the incident wave field, equation (3) reduces to the following form:

$$
\begin{aligned}
& \Sigma_{\mathrm{A}} \nabla \bullet\left(\mathbf{E}^{\mathbf{i}}-\mathbf{E}^{\mathbf{r}}\right)=\nabla \Sigma_{\mathrm{P}} \bullet\left(\mathbf{E}^{\mathbf{i}}-\mathbf{E}^{\mathbf{r}}\right) \\
&+\Sigma_{\mathrm{P}} \nabla \bullet\left(\mathbf{E}^{\mathbf{i}}-\mathbf{E}^{\mathbf{r}}\right)
\end{aligned}
$$

Letting both the conductivity gradient and the incident wave field lie in the $\mathrm{X}$ direction, equation (6) becomes a onedimensional equation that can be written as:

$$
\frac{d E^{r}}{d x}+\frac{\frac{d \Sigma_{P}}{d x}}{\Sigma_{H}+\Sigma_{P}} E^{r}=\frac{\Sigma_{A}-\Sigma_{P}}{\Sigma_{A}+\Sigma_{P}} \frac{d E^{i}}{d x}-\frac{\frac{d \Sigma_{P}}{d x}}{\Sigma_{A}+\Sigma_{P}} E^{i}
$$

The general solution to equation (7) is:

$$
E^{r}=\frac{d}{\Sigma_{A}+\Sigma_{P}}\left\{\int\left[\left(\Sigma_{A}-\Sigma_{P}\right) d E^{i}-E^{i} d \Sigma_{P}\right]+c\right\}
$$

where $d$ and $\mathrm{c}$ are integral constants. Letting $\left(\Sigma_{\mathrm{A}}-\Sigma_{\mathrm{P}}\right) \mathrm{E}^{\mathrm{i}}=$ $\mathrm{E}^{\mathrm{i}^{*}}$, we have the solution for $\mathrm{E}^{\mathrm{r}}$

$$
E^{r}=\frac{d}{\Sigma_{A}+\Sigma_{P}}\left(\Sigma_{A}-\Sigma_{P}\right) E^{i}+\frac{c^{*}}{\Sigma_{A}+\Sigma_{P}}
$$

Since $\mathrm{E}^{\mathrm{r}}=0$ when $\mathrm{E}^{\mathrm{i}}=0$, and $\mathrm{E}^{\mathrm{r}}=\frac{\Sigma_{\mathrm{A}}-\Sigma_{\mathrm{P}}}{\Sigma_{\mathrm{A}}+\Sigma_{\mathrm{P}}} \mathrm{E}^{\mathrm{i}}$ when $\nabla \Sigma_{\mathrm{P}}=$ 0 , this then requires that $\mathrm{c}^{*}$ must be zero and $\mathrm{d}$ must be 1 .

Now we obtain the reflection coefficient for the case in which the conductivity gradient is parallel (or anti-parallel) to the incident wave field,

$$
\mathrm{R}^{*}=\frac{\mathrm{E}^{\mathrm{r}}}{\mathrm{E}^{\mathrm{i}}}=\frac{\Sigma_{\mathrm{A}}-\Sigma_{\mathrm{P}}(\mathrm{x})}{\Sigma_{\mathrm{A}}+\Sigma_{\mathrm{P}}(\mathrm{x})}
$$

When the nonuniformity of the ionospheric conductivity is along the direction of the incident wave field, the reflection coefficient takes the same form as that of a homogeneous ionosphere, except that $R^{*}$ is now a function of space instead of being a constant. It can be seen that for this specific 


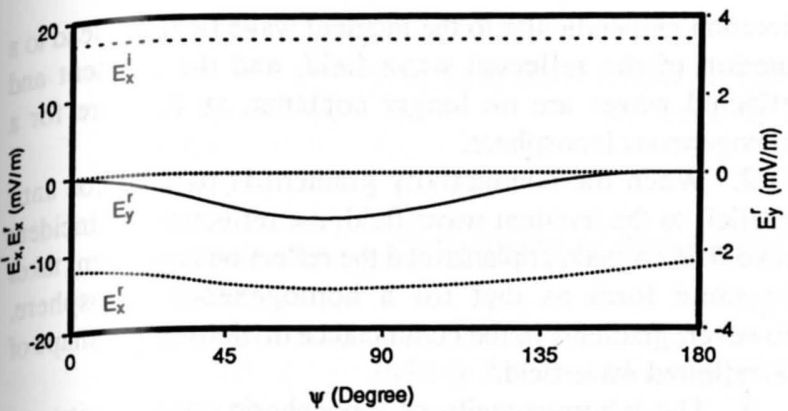

Fig. 1. Relationship of the wave fields to the angle $\psi$. The dashed line denotes the incident wave field which is in the $X$ direction, the dotted line denotes the $\mathrm{X}$ component of the reflected wave field, and the solid line denotes the $Y$ component of the reflected wave field. The left coordinate is for the $\mathrm{X}$ components of the wave fields and the right coordinate is for the Y component of the reflected wave field.

situation, $\mathrm{E}^{\mathrm{i}}$ and $\mathrm{E}^{\mathrm{r}}$ are still coplanar, and the inhomogeneity of the ionospheric conductivity only deforms the shape of the reflected wave field. The amplitude and phase of the reflected wave field are still determined by the ratio of the Alfvén conductivity to the Pedersen conductance.

But there does exist an important difference between equations (5) and (10), which is the ionosphere-originated field-aligned current as discussed in the previous section. For example, when we assume an uniform $\mathrm{E}^{\mathrm{i}}$, we obtain an uniform $\mathrm{E}^{\mathrm{r}}$ from equation (5), but we can obtain a nonuniform $\mathrm{E}^{\mathrm{r}}$ from equation (10). This means that with a uniform $\mathrm{E}^{\mathrm{i}}$, the reflected wave from a horizontally inhomogeneous ionosphere can carry a field-aligned current and this current has its source in the ionosphere.

\section{3. $\nabla \Sigma_{P} \neq 0, \psi \neq 0$}

In the general situation, equation (3) can no longer be reduced to a one-dimensional equation and we can only solve the equation numerically. In the following we will vary one parameter at a time to study how $\Sigma_{\mathrm{P}}, \nabla \Sigma_{\mathrm{P}}, \psi$, and R affect the Alfvén wave reflection at a horizontally inhomogeneous ionosphere.

Figure 1 shows the relationship of the wave fields to the angle $\psi$. In the calculations, the Pedersen conductance at the observation point $\Sigma_{\mathrm{P}}^{0}=4 \mathrm{mho}$, the ratio $\mathrm{R}=\left(\Sigma_{\mathrm{H}} / \Sigma_{\mathrm{P}}\right)=1.5$, $\left|\nabla \Sigma_{\mathrm{P}}\right|=0.666 \times 10^{-} \mathrm{P}_{5} \mathrm{mho} / \mathrm{m}$, and they remained constant as $\psi$ varied. It can be seen that when $\psi=0^{\circ}$ or $180^{\circ}$, the incident

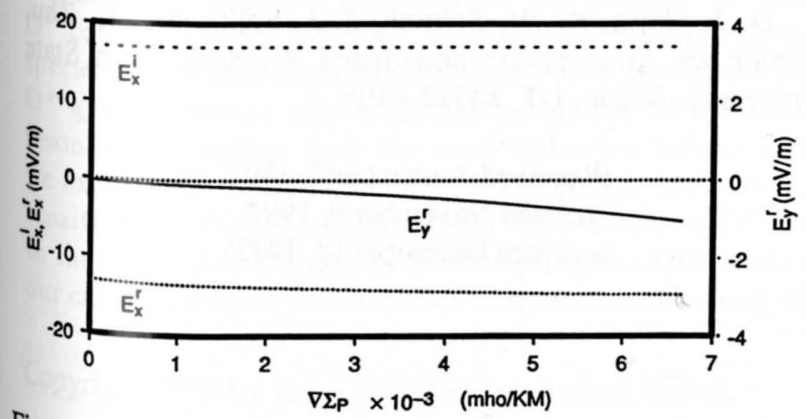

Fig. 2. Relationship between wave fields and conductivity gradient. This figure uses the same format as Figure 1.

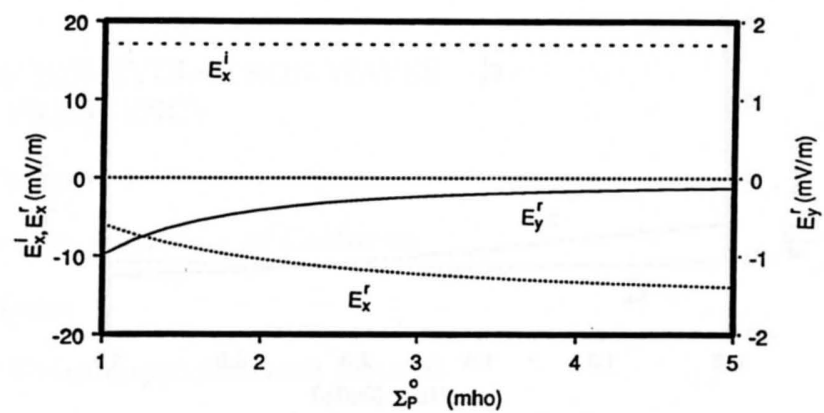

Fig. 3. Relationship between wave fields and local Pedersen conductivity. This figure uses the same format as Figure 1.

and reflected wave fields are coplanar, which is consistent with the analytical solution in the previous subsection. The influence of the Hall current on the reflection feature increases as $\psi$ approaches $90^{\circ}$ and the $\mathrm{Y}$ component of the reflected wave field becomes larger. The maximum rotation of the reflected wave field is achieved when $\psi$ is close to $90^{\circ}$. To satisfy the current continuity in the ionosphere, there is a corresponding change of the $\mathrm{X}$ component of the reflected wave. The slight asymmetry of the reflected wave field around $90^{\circ}$ is because the total electric field rotates to an angle with respect to the $\mathrm{X}$ axis and the maximum influence of the Hall current does not occur at $\psi=90^{\circ}$.

Figure 2 shows the relationship between the wave fields and the conductivity gradient. In the calculations, $\Sigma_{\mathrm{P}}^{0}=4 \mathrm{mho}$, $\mathrm{R}=1.5, \psi=90^{\circ}$, and they remain constant as $\nabla \Sigma_{\mathrm{P}}$ is varied. As indicated, there is no rotation of the reflected wave field when the conductivity is uniform, and a larger conductivity gradient leads to a larger rotation of the reflected wave field. Figure 3 shows the relationship between the wave fields and the local Pedersen conductivity at the observation point. In the calculations, $\mathrm{R}=0.5,\left|\nabla \Sigma_{\mathrm{P}}\right|=1.8 \times 10^{-6} \mathrm{mho} / \mathrm{m}$, and $\psi=$ $90^{\circ}$. It can be seen that as $\Sigma_{\mathrm{p}}^{0}$ increases, the X component of the reflected wave field increases and the rotation of the reflected wave field becomes weaker. Finally, Figure 4 shows the relationship between the wave fields and the conductivity ratio $R$. In the calculations, $\Sigma_{\mathrm{P}}^{0}=2 \mathrm{mho},\left|\nabla \Sigma_{\mathrm{P}}\right|=0.5 \times 10^{-5}$ $\mathrm{mho} / \mathrm{m}$, and $\psi=90^{\circ}$. This shows that as $\mathrm{R}$ increases, the rotation of the reflected wave field increases. This indicates that with a larger $\mathrm{R}$, the Hall current plays a stronger role in determining the reflection feature of the waves and leads to a stronger rotation of the electric fields.

\section{Implications for Ionospheric Phenomena}

The theoretical results presented in the previous sections have their implications for some ionospheric phenomena.

The region of the Harang discontinuity is a very special region in the ionosphere where the electric field makes a sudden rotation and convection appears to be distorted. Previous work suggests that this is due to the specific configuration of the magnetospheric convection [e.g., Erickson et al., 1991]. Based on the results in the previous sections, we propose a possible ionospheric explanation for the occurrence of the Harang discontinuity. Assuming that the initial magnetospheric convection carried by Alfvén waves is a symmetric two-cell convection pattern, then in the auroral oval near midnight the incident wave field is almost perpendicular 


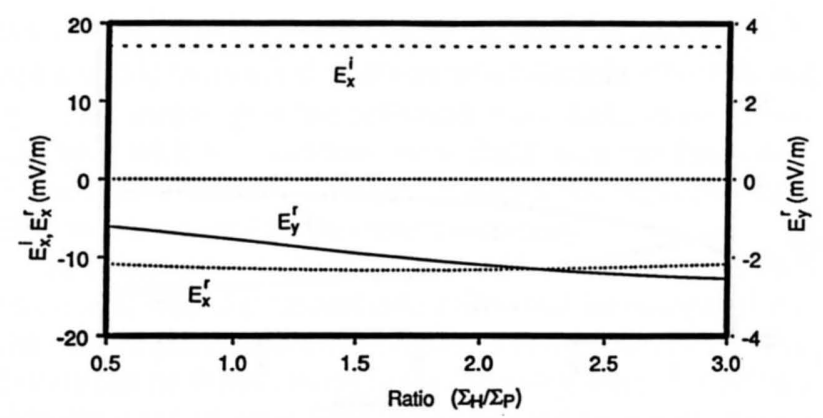

Fig. 4. Relationship between wave fields and ratio R. This figure uses the same format as Figure 1.

to the conductivity gradient caused by diffuse auroral precipitation. Also, the conductivity gradient and the ratio of the Hall and Pedersen conductivities in that specific region are larger than those in other polar regions. Based on our results, a strong rotation of the reflected wave field must occur, which could lead to the distortion of the convection. Our results show that for an incident wave field $E^{i}$ of $10 \mathrm{mv} / \mathrm{m}$, a Pedersen conductivity gradient $\nabla \Sigma_{\mathrm{P}}$ of $1.8 \times 10^{-5} \mathrm{mho} / \mathrm{m}$, a conductivity ratio $R=1$, and a local conductivity $\Sigma_{\mathrm{P}}=1 \mathrm{mho}$, the convection electric field can rotate by as much as $36^{\circ}$.

Sun-aligned arcs in the polar cap are the focus of intense study. Many observations have shown that a strong shear flow is often associated with these arcs, but substantial flows across the arcs have also been reported [Valladares and Carlson, 1991]. We suggest that the occurrence of such a cross flow might also be due to the rotation of the wave field caused by the horizontal inhomogeneity of the ionospheric conductivity since, in most cases, the direction of the electric fields associated with the shear flows have a large angle with respect to the direction of the background conductivity gradient in the polar cap. The results from a typical case run in which the maximum intensity of the shear flow is $340 \mathrm{~m} / \mathrm{s}$ and the conductivity ratio $R=0.7$, show that the cross flow for the winter case, for which the Hall conductivity gradient, $\left|\nabla \Sigma_{\mathrm{H}}\right|=$ $0.9 \times 10^{-6} \mathrm{mho} / \mathrm{m}$ and the local Hall conductivity $\Sigma_{\mathrm{H}}=0.5$ mho, is around $25 \mathrm{~m} / \mathrm{s}$ and that for the summer case, for which $\left|\nabla \Sigma_{\mathrm{H}}\right|=2.1 \times 10^{-6} \mathrm{mho} / \mathrm{m}$ and $\Sigma_{\mathrm{H}}=4.5 \mathrm{mho}$, is around $1.2 \mathrm{~m} / \mathrm{s}$. Note that the above estimations are based on a single reflection of the waves. The magnitudes of the cross flows can be further changed when multiple wave bouncing and conductivity enhancements are included.

\section{Summary}

The reflection of Alfvén waves at a horizontally inhomogeneous ionosphere has been studied in the situation where the Alfvén speed above the ionosphere is assumed to be uniform and the ionosphere is treated as a height-integrated conducting slab. The main results of the study can be summarized as follows:

1. A nonuniformity of the ionospheric conductivity in the direction perpendicular to the incident wave field can lead to a rotation of the reflected wave field, and the incident and reflected waves are no longer coplanar, as they are for a homogeneous ionosphere.

2. When the conductivity gradient is parallel (or antiparallel) to the incident wave field, the reflected and incident wave fields remain coplanar and the reflection coefficient takes the same form as that for a homogeneous ionosphere. However, gradients in the conductance do deform the shape of the reflected wave field.

3. The inhomogeneity of ionospheric conductivity can lead to field-aligned currents that originate in the ionosphere.

4. Increasing the gradient of conductivity in the direction perpendicular to the incident wave field, increasing the ratio of the Hall and Pedersen conductivities, and lowering the conductivity all lead to a larger rotation of the reflected wave field.

The implications of our theoretical results for some ionospheric phenomena have been discussed. For typical conditions found in the auroral and high-latitude ionosphere, these results imply a rotation of the reflected wave fields in the range of from a few to $40^{\circ}$.

Acknowledgments. This research was supported by NASA grant NAG5-1484 and NSF grant ATM-89-13230 to Utah State University.

\section{References}

Alfvén, H., and C.G. Falthammar, Cosmical electrodynamics, Fundamental Principles, Clarendon, Oxford, 1963.

Erickson, G. M., R. W. Spiro, and R. A. Wolf, The physics of the Harang discontinuity, J. Geophys. Res., 96, 1633, 1991.

Knudsen, D. J., M. C. Kelley, and J. F. Vickery, Alfvén waves in the auroral ionosphere: A numerical model compared with measurements, J. Geophys. Res., 97, 77, 1992.

Lysak, R. L., Feedback instabilities of the ionospheric resonant cavity, J. Geophys. Res., 96, 1553, 1991.

Mallinckrodt, A. J., and C. W. Carlson, Relations between transverse electric fields and field-aligned currents, $J$. Geophys. Res., 83, 1426, 1978.

Valladares, C. E., and H. C. Carlson, Jr., The electrodynamic, thermal, and energetic character of intense sun-aligned arcs in the polar cap, J. Geophys. Res., 96, 1379, 1991.

D. J. Crain, R. W. Schunk, J. J. Sojka, and L. Zhu, Center for Atmospheric and Space Sciences, Utah State University, Logan, UT 84322-4405.

(Received September 2, 1992; revised November 9, 1992; accepted December 14, 1992) 